Punkt nicht allzu naiv sein sollte). Vielleicht liegt einem die Praxis auch mehr als die Wissenschaft, wie es etwa bei Holger Rother, heute Berater bei der Management Search GmbH in Frankfurt, war. Er brauchte für sein Mathematikstudium stolze 18 Semester. „In der Schule war für mich nichts schöner als der Mathe Leistungskurs", sagt er, "das Studium war für mich nur noch trocken und von der Welt losgelöst." Parallel zum Studium gründete er ein eigenes Unternehmen, eine Art Studienkreis, wo er Professoren und Studenten an Nachhilfeschüler vermittelte. Heute vermittelt er Führungskräfte quer durch die Republik. Die guten Management-Eigenschaften von Mathematikern, die Ulrich Hirsch aufführt, würden „schmeicheln“, sagt Rother ein wenig ironisch. Im Studium galt er gerade wegen seiner unternehmerischen Ader als Außenseiter.

Woran erkennt man, ob ein Schüler für das Mathematik-Studium geeignet ist? Jens Frehse, Pro- fessor an der Universität Bonn, hat dies im Rheinischen Merkur so beantwortet: „Wenn er sich allein mit einem Mathebuch beschäftigt und Spaß hat, über ihm zu brüten." - Wie erkennt man nun, ob ein Student für einen Management-Beruf geeignet ist? Ganz einfach: ihn für zwei, drei Monate die Bücher beiseite legen und die Praxis ausprobieren lassen. Es könnte ja sein, daß er nicht nur ein guter Student, sondern auch ein Organisations- und Kommunikationstalent ist. Vielleicht findet er sich einige Jahre später an den Schaltstellen in Politik, Wirtschaft oder Verwaltung wieder - als Mathematiker unter den Juristen und anderen Exoten.

\section{Adresse des Autors}

Vasco Alexander Schmidt

Stubenrauchstraße 40

12161 Berlin-Friedenau

vasco@math.fu-berlin.de

\title{
Mathematiker im Management
}

\section{Ein Interview mit Dr. Gerhard Rupprecht, Vorstandsvorsitzender der Allianz Leben}

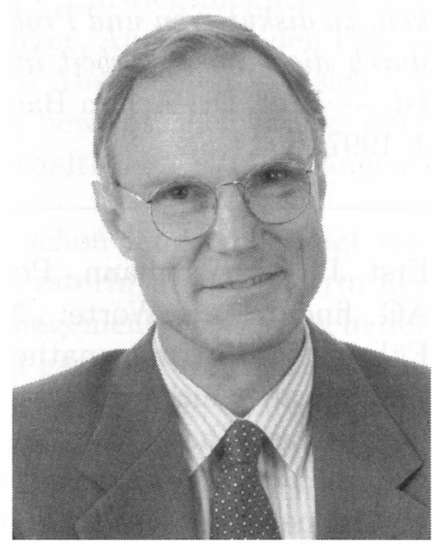

Dr. Gerhard Rupprecht, Jahrgang 1948, arbeitete nach seinem Mathematik-Studium zunächst als wissenschaftlicher Angestellter an der Universität Stuttgart. 1978 wurde er Systemplaner bei der Standard Elektrik Lorenz AG (SEL) und wechselte 1979 zur Allianz Lebensversicherungs-AG, wo er als Versicherungsmathematiker tätig war. Seit 1989 ist er Vorstandsmitglied, seit 1991 Vorsitzender des Vorstands von Allianz Leben, zudem sitzt er im Vorstand der Allianz AG.

In Ihrer Festrede zum 20-jährigen Bestehen des Ulmer Studiengangs Wirtschaftsmathematik haben Sie den Studiengang als die gute Umsetzung eines universitären Marketingkonzeptes gelobt. Darf man im Umkehrschluß behaupten, die Universitäten würden ihre Studenten in der Regel am Markt vorbei ausbilden?

Nein, so habe ich das nicht gemeint. Im Grunde bin ich davon überzeugt, daß auch ein reines Mathematikstudium für Aufgaben in der Wirtschaft qualifiziert. Nur fällt es den Mathematikern häufig schwer, den Personalabteilungen klar zu machen, daß Mathe- matik praktische Anwendungen hat. Deshalb sind die Mathematiker in gewisser Weise zu der Notlösung gezwungen, durch die Etikettierung des Studiengangs bereits zu demonstrieren, daß es heute zum Beispiel in den Wirtschaftswissenschaften signifikante Anwendungen von Mathematik gibt. Dort hat sie inzwischen eine Bedeutung, die über das Rechnen und Buchhalten hinausgeht. Mittlerweile finden tieferliegende mathematische Theorien Anwendung.

Wirtschaftstheoretiker gehen davon aus, daß wir einem neuen, dem sogenannten sechsten KondratieffZyklus entgegengehen, daß nach Stahl und Öl heute 
auch Information als Rohstoff aufzufassen ist. Steigt dadurch auch die Bedeutung der Mathematik?

Die Mathematik hatte schon immer eine große Bedeutung für die Weiterentwicklung der Wirtschaft. Man würde den historischen Einfluß der Mathematik erheblich unterschätzen, wenn man ihr nur im Zusammenhang mit der Informationstechnik eine spezifische Rolle zuweisen würde. Im Bankwesen hat es aber tatsächlich einen gewissen Quantensprung gegeben. Die Finanzinstrumente, die man heute braucht, sind im Grunde nichts anderes als mathematische Konstrukte. Die berühmte Black-Scholes-Formel ist nicht nur ein mathematischer Algorithmus, nach ihr richtet sich letztlich der Markt. Sie ist ein mathematisches Bewertungsverfahren, das das Wertsystem selbst prägt, eine unglaubliche normative Kraft. Man führt den Börsencrash 1987 unter anderem darauf zurück, daß Computer aufgrund von einprogrammierten Algorithmen in einer breiten Welle Verkaufssignale gegeben haben, und kein Mensch mehr mitentschieden hat. Als dann die Kurse in den Keller gingen, hatte das ganz reale Wirkungen. Manche Leute haben ihren Arbeitsplatz verloren, andere sind arm geworden. Das zeigt die Gefahr, daß mathematische Konstrukte im richtigen Leben kritiklos - das heißt unmathematisch - angewendet werden. Es besteht oft ein blinder Glaube in die Fähigkeit, durch ein normatives Konstrukt etwas zu beschreiben, das noch nicht Realität ist - und die Kapitalmärkte werden ja bestimmt durch Zukunftserwartungen. Insofern ist die Mathematik mit Chancen und Risiken in eine neue Rolle hineingewachsen, in der sie nicht mehr Realität beschreibt, sondern umgekehrt die Realität prägt.

In vielen Unternehmen werden mittlerweile Naturwissenschaftler und Mathematiker im Marketing eingesetzt. Produkte und Abläufe, die vermittelt werden müssen, sind oft so kompliziert geworden, daß man auf logisch geschulte Mitarbeiter setzt. Auch bei der Allianz Leben arbeiten Mathematiker im Vertrieb. Gab es hier eine Entwicklung oder ist der Bereich für Mathematiker bei Ihnen klassisch?

Das muß man differenziert sehen. Daß bei uns auch Mathematiker im Marketing tätig sind - besonders viele sind es nicht - , liegt daran, daß wir in der Lebensversicherung traditionell außerordentlich viele Mathematiker beschäftigen. Hier müssen über 30 Jahre Annahmen über Sterblichkeitsentwicklung, über Zinsentwicklung, über Kostenentwicklung getroffen werden, um Leistungen, zu denen sich das Unternehmen schon heute verpflichtet, die aber erst in 30 Jahren fällig werden, mit einem Wert zu versehen. Eine schwierige Frage: Was ist der Wert der Garantie, in 30 Jahren 1000 Mark gezahlt zu bekommen, geschehe was wolle? Die mathematischen
Handwerkszeuge helfen bei diesem Problem, doch besteht die Lebensversicherungs-Mathematik im Grunde aus den vier Grundrechenarten - Mathematik als Tool ist fast irrelevant. Wichtig ist der gedankliche Ansatz, die Bildung des abstrakten Modells. Das ist etwas, was Mathematiker lernen und deshalb in der Praxis gut umsetzen können. Diese Fähigkeit der Modellbildung braucht man in vielen Gebieten. Ich glaube, daß in den heutigen Unternehmen, deren Arbeitsvorgänge in den vergangenen Jahren zweifellos viel komplexer geworden sind, der reine Praktikeransatz nicht mehr erfolgreich ist. Aus reinen Effizienzgründen ist es entscheidend, daß man in den entsprechenden Bereichen Leute hat, die Modelle bilden und die Prioritäten richtig setzen können. Die Mathematik ist ja die Kunst des geschickten Vernachlässigens, und das ist natürlich auch in den Unternehemen heute wichtig, weil die Dinge so verästelt sind. Auf der anderen Seite kommen wir in Deutschland aufgrund der immer stärkeren Querverbindungen der Wirtschaftszweige und Bereiche untereinander immer weiter weg von der Frage, welches Fach jemand studiert hat. Es geht um die Überzeugung: Dies ist ein guter Kopf, der hat Denken gelernt, der macht das dann. Ob er oder sie Altphilologe ist oder Mathematiker, ist vielleicht gar nicht das Entscheidende.

Sie stellen sicher trotzdem mehr Mathematiker als Altphilologen, Philosophen oder Historiker ein.

Natürlich. Ein Unternehmen wird immer noch und sicher mit. Recht vermuten, daß ein Mathematiker mit größerer Wahrscheinlichkeit die erforderlichen Fähigkeiten mitbringt als ein Absolvent einer anderen Studienrichtung. Es gibt noch einen weiteren Vorzug, den ein Mathematiker hat, wenn er sich bei einer Versicherung, insbesondere bei einer Lebensversicherung, bewirbt: er trifft auf Leute, die auch Mathematiker sind. Und ein Mathematiker stellt gern Mathematiker ein. Nur darf man sich nicht täuschen. Auch ein Jurist stellt gern einen Juristen ein.

Wenn der Einstieg geschafft ist, werden aus Mathematikern gute Manager?

Ich glaube nicht, daß man diese Frage mit ja oder nein beantworten kann. Die Frage des guten Managers ist vor allem die des Umgangs mit Menschen. Ob nun Mathematiker aufgrund ihrer Ausbildung dafür prädestiniert sind, weiß ich nicht. Das ist, glaube ich, eine Eigenschaft, die relativ unabhängig von dem Ausbildungshintergrund und den abstrakten Fähigkeiten ist.

Prof. Hirsch hat in einem Vortrag einige Eigenschaften, die man Mathematikern zuschreibt, aufgezählt, 
die hervorragend zum Manager-Beruf passen. Darunter Beharrlichkeit, Intuition, Reduktion von Komplexität, Offenheit, ständiges Weiterlernen, Konzentrationsfähigkeit, Strategisches Denken. Ist es also doch nützlich, Mathematiker zu sein?

Ja, sicher. Nur glaube ich nicht, daß sich Mathematiker einbilden sollten, allein durch ihr Studium für das Management prädestiniert zu sein. Ein guter Jurist besitzt auch logisches Denken. Die Meinung, Mathematik und Jura seien so arg unterschiedlich, trifft vielleicht gar nicht zu. Ich glaube, es gibt Juristen, die hätten auch Mathematik studieren können und umgekehrt. Vielleicht nicht auf einem Niveau, um Weltstars zu werden, aber auf dem wissenschaftlichen Niveau, das im Management benötigt wird.

Gibt es umgekehrt Dinge im Mathematik-Studium, die hinderlich sind?

Es gibt einen großen Teil von Mathematikern, denen es an der vorhin erwähnten Offenheit mangelt, die die Dinge nur mathematisch sehen und vergessen, daß sich Management-Entscheidungen oder Entscheidungen im richtigen Leben, die wirklich schwierig sind, gerade nicht zweiwertig behandeln lassen. Die schwierigen Entscheidungen im Wirtschaftsleben sind immer die, wo ja oder nein nicht feststehen, wo man gerade nicht „q.e.d." drunterschreiben kann. Alle Dinge, die mathematisch geklärt werden können, werden selbstverständlich auf der Arbeitsebene abgehandelt. Die eigentliche Kunst liegt im Umgang mit den Unsicherheiten und außermathematischen Grauzonen. Damit kommen viele Mathematiker aufgrund ihrer Ausbildung nicht zurecht, auch wenn die guten Mathematiker damit durchaus umgehen können.

Haben Sie eine Botschaft an die Universitäten? Was sollte sich an der Ausbildung geändert werden?

Meine Botschaft an die Mathematiker an den Universitäten ist die folgende: Sie sollen sich nicht beeindrucken lassen vom falsch verstandenen Ruf nach Praxisnähe. Das ist der leichte Ausweg. Die Mathematiker müssen im Gegenteil darauf beharren, daß an den Universitäten harte mathematische Bretter gebohrt werden, die wirklich - wie vorhin zitiert wurde - die Beharrlichkeit fördern, ein Problem anzugehen und zu Ende zu bringen. Manchmal habe ich eher den Eindruck, daß die Universitäten glauben, sie sollen der Vorstellung nachgeben, die Mathematik sei am nächsten Tag umsatzwirksam anzuwenden. Die Praxis können die Unternehmen besser, die Universitäten sollen sich nicht einbilden, in der Formulierung praktischer Probleme mit Unternehmen konkurrieren zu wollen. Es sollte ihnen vielmehr klar sein, daß ihr Auftrag ist, Spitzenforschung zu leisten - soweit die Studenten bereit und in der Lage sind zu folgen. Ich bin überzeugt, daß mathemati- sche Spitzenforschung eben gerade die Negativstereotypen der Abgeschlossenheit, der Nicht-Offenheit und des Nicht-Trauens überwindet. Deshalb meine Überzeugung: Mathematiker, die dort ausgebildet werden, sind in der Praxis mindestens genauso nützlich wie andere Absolventen.

Ihr großes Engagement für die Oberwolfach-Stiftung und das Lob für den Studiengang Wirtschaftsmathematik in Ulm widersprachen sich demnach nicht.

Überhaupt nicht. Das ist einfach eine Frage des Marketings. Ich glaube, man kann Zahlentheorie machen und hinterher ein Unternehmen führen. Man darf sich nur nicht einbilden, daß Unternehmensführung Zahlentheorie ist.

Ihre Botschaft scheint vor allem eine für die Hochschullehrer zu sein. Wie lautet ihre Botschaft an die Studierenden? Soll man Hochschulabsolventen ermutigen, bei der Jobsuche auch in Bereiche zu gehen, die für Mathematiker nicht klassisch sind?

Unbedingt. Sie werden sogar auffallen, wenn sie sich für etwas bewerben, für das sich Mathematiker normalerweise nicht bewerben. Sie müssen nur selbst wissen, ob sie der Typ von Mathematiker sind, der das machen kann. Zweifellos gibt es einen großen Teil von Mathematikabsolventen, die in einer mathematiknahen Tätigkeit bleiben sollten. Man muß zugeben, daß es den Typus von Mathematiker gibt, dem schlicht und einfach die Kommunikation mit Leuten außerhalb der Mathematik schwerfällt. In einem Unternehmen Karriere zu machen, hängt heutzutage im hohen Maße und auch mit Recht davon ab, inwieweit man in der Lage ist, die eigenen Fähigkeiten und Erkenntnisse anderen mitzuteilen. Dinge in einer Art und Weise mitzuteilen, die erst in drei Generationen richtig verstehen werden kann, mag in der forschenden Mathematik noch angehen - obwohl ich glaube, daß gerade die großen Mathematiker gut schreiben können, das unterscheidet ja die großen von den nicht so großen Mathematikern. In einem Unternehmen sind Dinge, die nicht mitgeteilt werden können, wertlos. Das Mitteilungsvermögen von Mathematikern in reinem Deutsch, ohne Formeln, ist etwas, das auch an der Universität — zum Beispiel in Seminaren - mehr geschult werden sollte.

Viele Studierende haben - so ist mein Eindruck wenig außerhalb der Universitäten gesehen. Praktika könnten helfen. Soll man sie in die Studienordnung aufnehmen?

Das ist ein ganz wesentlicher Punkt. Gerade ein Mathematiker sollte unbedingt in seiner Studienzeit Praktika machen. Ich glaube allerdings nicht, daß es notwendig ist, Praktika auszuwählen, die unmittelbar zur späteren Berufswahl hinführen. Auch hier muß man sich von der Idee freimachen zu wissen, 
welchen Beruf man in drei Jahren wirklich ausübt und dann völlig verunsichert zu sein, wenn es nicht funktioniert.

\section{Welche Praktika sind günstig?}

Sehr nützlich - mal abgesehen von den Inhalten ist immer das Ausland, und das aus rein pragmatischen Gründen. Heutzutage wird von vielen Unternehmen Internationalität verlangt, und da ist es gutes Personalmarketing, wenn man in den eigenen Bewerbungsunterlagen einen Auslandsaufenthalt nachweisen kann. Aber es schult auch - und das ist, glaube ich, bei Mathematikern manchmal notwendig das Zurechtkommen in unsortierten Welten. Die Mathematik ist immer — vielleicht nur vermeintlich wohlsortiert. Da sollten sich Mathematiker Situationen aussetzen, die sozusagen noch theoriefrei sind. Im Grunde werden sie genau dort gebraucht. Deshalb ist es nicht gut, ein Praktikum zu machen, wo man sozusagen nur auf wesentlich trivialerem theoretischem Niveau dasselbe macht wie im Studium. Eine Bilanz zu schreiben - wieder ein abstraktes Konstrukt, nur statt mit Differentialgleichungen mit den Grundrechenarten - wird nichts bringen. Ich würde daher Praktika in Bereichen vorschlagen, die produktionsnah und kundennah sind, Marketing zum Beispiel. Hier lernen Mathematiker, mit Leuten zu kommunizieren, die völlig anders denken als sie.

Es gibt das Vorurteil gegenüber Mathematikern, daß sie zu theoretisch und unverständlich sind, und ein wenig skurril.

Ich war zuerst bei einem nachrichtentechnischen Unternehmen, der SEL in Stuttgart, beschäftigt. Ich übertreibe jetzt, um es plakativer zu machen: Dort gab es vor allem Nachrichtentechniker. Nun müssen Nachrichtentechniker höhere Mathematik an der Universität belegen, und je nachdem wie die Vorlesung war, haben sie ein mehr oder weniger ausgeprägtes Vorurteil gegenüber Mathematikern. Dem Mathematiker, der sich unter den Ingenieuren behaupten will, gereicht das oft nicht zum Guten. Dort merkt dann man als Mathematiker, daß man sich auch erstmal etablieren muß als jemand, der etwas Nützliches zustande bringt, und zwar gerade weil er den theoretischen Ansatz wählt, um die Praxis zu vereinfachen. Als Mathematiker läuft man üblicherweise Gefahr, diskriminiert zu werden, indem gesagt wird: Ach, das ist ja nur ein Theoretiker.

Sich bewähren zu müssen, heißt aber auch, daß man sich bemerkbar machen kann.

Es gibt noch einen anderen Effekt: Bei aller Skurrilität, die Mathematikern angedichtet wird, schwingt natürlich auch Ehrfurcht mit. Dazu kommt eine gewisse Gläubigkeit in die Funktionstüchtigkeit der Mathematik, die nicht im Widerspruch zu der Mei- nung steht, die Mathematiker seien alle ein bißchen $\mathrm{zu}$ theoretisch und $\mathrm{zu}$ nichts $\mathrm{zu}$ gebrauchen. Im Gegenteil, wie vorhin erwähnt, ist die Gläubigkeit an Kapitalmärkten ganz unglaublich. Insofern glaube ich schon, daß Mathematiker, wenn sie es richtig machen, heute gute Chancen haben. Anders als noch vor vielleicht 20 Jahren werden werden in Bereichen schon jetzt routinemäßig Mathematiker gesucht, die man dort früher nicht eingestellt hätte. Bei uns in der Kapitalanlage sind heute viele Mathematiker beschäftigt. Früher waren im Kapitalmarktbereich ausschließlich Juristen. In der Betriebswirtschaft, auch im Rechenwesen, sind bei uns mehr Mathematiker tätig als Betriebswirte. Drei von unseren sechs Vorständen sind Mathematiker. Der Vorsitzende einer Lebensversicherungsgesellschaft braucht natürlich nicht von Natur aus Mathematiker zu sein, natürlich nicht. Daß unser Produktentwickler ein Mathematiker ist, eben weil die Lebensversicherung ein sehr abstraktes finanztechnisches Instrument darstellt, ist schon naheliegender. Daß aber der EDVChef Mathematiker ist und kein Informatiker oder Betriebswirt zeigt eben, daß dort der Mathematiker in Konkurrenz mit anderen Fachleuten sehr gut mithalten kann.

Wenn sie von Mathematikern sprechen, denken sie dann an einen Wirtschaftsmathematiker? Stellen Sie auch Topologen ein oder lieber jemanden, der etwas Angewandteres wie Analysis studiert hat?

Die Prämisse, die ich jetzt setze, ist eigentlich unzulässig, weil sie selten zutrifft: Wenn es sonst keinerlei Unterscheidungsmerkmale gibt zwischen zwei Bewerbern - und es gibt auch keine Unterschiede in der wissenschaftlichen Qualität -, dann würden wir jemanden, der Wahrscheinlichkeitstheorie gemacht hat, jemandem vorziehen, der Topologie gemacht hat. Nur glaube ich, daß dieses Unterscheidungsmerkmal angesichts der vielen anderen, die vorher kommen, eher irrelevant ist.

Zur Zeit berichten die Zeitungen auf ihren Berufsund Karriereseiten, daß einem Wirtschaftsmathematiker drei offene Stellen gegenüberstehen. Was passiert mit den anderen zwei Drittel der Stellen, wer kommt da zum Zug?

Meine Meinung ist, daß die Lage für Mathematiker schlechthin gut ist und daß eine Stelle, die für einen Wirtschaftsmathematiker ausgeschrieben ist, sich für jeden Mathematiker anbietet und jeder Mathematiker eingestellt wird, der im Vorstellungsgespräch und der Präsentation seiner Person einfach rüberbringen kann, daß er in einer im Grunde unstrukturierten Welt im Wirtschaftsleben mit seiner Theorie etwas machen kann. Ich glaube, die Ausschreibung für Wirtschaftsmathematiker soll nur signalisieren, daß dort niemand gesucht wird, der nur hochspezialisierte 
mathematische Arbeit leistet. Ein Mathematiker, der bereit ist, sein Mathematikstudium sozusagen als ein Training seiner Grundfähigkeiten anzusehen, der sollte sich nicht scheuen, sich auf eine Stellenanzeige für Wirtschaftsmathematiker zu bewerben. Nur jemand, der in seinem Berufsleben wirklich harte Mathematik machen möchte, dessen Interesse im mathematischen Problem selbst liegt, der sollte sich auf so eine Anzeige nie bewerben. Denn die Schwierigkeit, die Leute, die als Wirtschaftsmathematiker gesucht werden, zu lösen haben, ist nicht das mathematische Problem. Der Wirtschaftsmathematiker wird benötigt, um das Problem der Mathematik zugänglich zu machen, damit das mathematische Instrumentarium ablaufen kann. Und er muß das Ergebnis interpretieren, damit es im Unternehmen umgesetzt werden kann. Er muß beurteilen können im Sinne von Verstehen.
Seine Kreativität ist an den Schnittstellen gefordert. Er muß wissen, was sich dazwischen abspielt, aber er muß in der Regel nichts Neues erfinden. Das sollen die Universitäten machen. Das ist gemeint mit der Stellenanzeige Wirtschaftsmathematiker. Ich glaube, es gibt viele Mathematiker, die durch diese Situation verunsichert sind, die glauben, daß sie in Unternehmen dafür bezahlt werden, daß sie im mathematischen Teil perfekt sind - es gibt einzelne solche Stellen, aber sie sind sehr vereinzelt. Die meisten Mathematiker werden dafür bezahlt, daß sie ein Problem der mathematischen Behandlung zugänglich machen und das Ergebnis kommunizieren können.

Vielen Dank für das Gespräch.

Die Fragen stellte Vasco Alexander Schmidt

\section{Mathematik in der DDR}

\section{von Helmut Koch}

Bei den folgenden Ausführungen geht es um die gesellschaftlichen Umstände von Forschung und Lehre in der Mathematik der DDR, wobei wir vorwiegend die Zeit von 1970 bis 1990 im Auge haben. Für die Berlin betreffende frühere Geschichte sowie die Überleitung in die Gegebenheiten der Mathematik des vereinten Deutschlands siehe [1].

Der vorliegende Bericht beruht im wesentlichen auf den Erinnerungen und Erfahrungen des Verfassers. Eine frühere Fassung wurde Mathematikern aller Universitäten der DDR zugeschickt. Die konstruktive Kritik dieser Kollegen habe ich in einer Reihe von Punkten eingearbeitet. In den meisten Fragen wurde das von mir Dargestellte bestätigt und ergänzt. Andererseits ergab sich aus den Zuschriften insgesamt kein vollständiger Überblick über alle Universitäten, so daß dieser Bericht in erster Linie die Verhältnisse im Mathematischen Institut der Akademie der Wissenschaften (AdW) der DDR schildert, an dem der Verfasser von 1959 bis 1991 gearbeitet hat. Die Verhältnisse an den Mathematischen Instituten der Universitäten erscheinen eher als Ergänzung. ${ }^{1}$

Unter allen Wissenschaften ist die Mathematik möglicherweise die am wenigsten vom Ost-WestAntagonismus abhängige. Dies gilt zumindest für die „kognitiven Aspekte", die in dieser Studie im
Vordergrund stehen. In allen Jahren der Teilung der Welt in eine westlich kapitalistische und eine östlich kommunistische gab es keine ideologischen Verständnisbarrieren, wo immer Mathematiker aus beiden Teilen zusammenkamen.

Ein kognitiver Aspekt, der in allen Wissenschaften auftritt, ist die gesellschaftspolitische Atmosphäre, in der Forschung stattfindet, stimuliert oder entmutigt wird. Dieser Aspekt wird im folgenden eine wesentliche Rolle spielen. Soweit es die DDR betrifft, kann diese gesellschaftspolitische Atmosphäre wohl nur von einem Betroffenen eingeschätzt werden, der zugleich in einer Stellung war, die ihm einen gewissen Einblick in wissenschaftspolitische Mechanismen gestattete. Hier sehe ich eine wesentliche Aufgabe dieses Berichtes.

Die hier vorliegende Fassung ist eine auf den Leserkreis der Mitteilungen der DMV zugeschnittene Kürzung einer Arbeit [2] im Rahmen der Ar-

1 Für ihre Mithilfe möchte ich mich insbesondere bei folgenden Kollegen herzlich bedanken: J. Böhm (Jena), H. Boseck (Greifswald), J. Eichhorn (Greifswald), H. Goering (Magdeburg), F. Hirzebruch (Bonn), R. Klötzler (Leipzig), E. Lanckau (Chemnitz) und P. Schenzel (Halle). 